\title{
Lycium barbarum polysaccharide protects against neurotoxicity via the Nrf2-HO-1 pathway
}

\author{
SHUMEI CAO ${ }^{1 *}$, JIANLONG DU ${ }^{2 *}$ and QIAOHONG $\mathrm{HEI}^{3}$ \\ ${ }^{1}$ Department of Anesthesiology, Xi'an No. 1 Hospital, Xi'an, Shaanxi 710002; \\ ${ }^{2}$ Department of Anesthesiology, No. 215 Hospital of Shaanxi Nuclear Industry, Xianyang, Shaanxi 712000; \\ ${ }^{3}$ Department of Anesthesiology, Xi'an High-Tech Hospital, Xi'an, Shaanxi 710075, P.R. China
}

Received August 1, 2016; Accepted June 8, 2017

DOI: 10.3892/etm.2017.5127

\begin{abstract}
The incidence of neurodegenerative diseases including Alzheimer's and Parkinson's disease has markedly increased over the past few decades. Oxidative stress is considered to be a common pathophysiological condition resulting in neurotoxicity. Lycium barbarum polysaccharide (LBP) is the major active component of Lycium barbarum L., which exhibit potent antioxidant activity. The current study investigated the neuroprotective effects of LBP in $\mathrm{H}_{2} \mathrm{O}_{2}$-treated PC12 cells in vitro and in $\mathrm{CoCl}_{2}$-treated rats in vivo. It was determined that LBP concentration-dependently reversed the $\mathrm{H}_{2} \mathrm{O}_{2}$-induced increase in reactive oxygen species (ROS) levels, decrease in cell viability, increase in TUNEL-stained cells, increase in caspase-3 and -9 activity and decrease in mitochondrial membrane potential, indicating the amelioration of mitochondrial apoptosis. Furthermore, LBP inhibited the $\mathrm{H}_{2} \mathrm{O}_{2}$-induced decrease in nuclear factor erythroid 2-related factor 2 (Nrf)2 and heme oxygenase (HO)-1 expression and binding of $\mathrm{Nrf} 2$ to the promoters of HO-1. Silencing of $\mathrm{Nrf} 2$ and inhibition of HO-1 by zinc protoporphyrin IX (ZnPP) reversed the protective effects of LBP against $\mathrm{H}_{2} \mathrm{O}_{2}$-resulted neurotoxicity in $\mathrm{PC} 12$ cells. In $\mathrm{CoCl}_{2}$-treated rats, it was demonstrated that LBP decreased brain tissue apoptosis, reduced the time spent by rats finding the platform site, decreased escape latencies and reduced the distance traveled to find the platform. In addition, $\mathrm{LBP}$ inhibited the $\mathrm{CoCl}_{2}$-induced decrease of $\mathrm{Nrf} 2$ and $\mathrm{HO}-1$ expression. Administration of $\mathrm{ZnPP}$ also suppressed the protective effects of $\mathrm{LBP}$ against $\mathrm{CoCl}_{2}$-resulted neurotoxicity in rats. Thus, the current study indicated that LBP exhibits protective effects against neurotoxicity by upregulating
\end{abstract}

Correspondence to: Dr Qiaohong Hei, Department of Anesthesiology, Xi'an High-Tech Hospital, 16 Tuanjie South Road, Xi'an, Shaanxi 710075, P.R. China

E-mail: qiaohong_hei301@126.com

*Contributed equally

Key words: Lycium barbarum polysaccharide, neurotoxicity, oxidative stress, nuclear factor erythroid 2-related factor 2, heme oxygenase-1
Nrf2/HO-1 signaling. These data may increase understanding regarding the neuroprotective activities of LBP.

\section{Introduction}

The incidence of neurodegenerative diseases, such as Alzheimer's disease, has increased dramatically over the past few decades $(1,2)$. Although the molecular mechanism of neurodegenerative diseases is not well understood, oxidative stress is considered to be a common pathophysiological condition that results in neurotoxicity $(3,4)$. The redox system consists of a free radical/reactive oxygen species (ROS)-generating system, oxidants and antioxidant systems (5). Following damage to the antioxidant system or an increase in ROS generation, the balance of the redox status may be disrupted, leading to the development of oxidative stress (5). The central nervous system is particularly susceptible to ROS-induced damage and numerous studies have demonstrated that oxidative stress is closely associated with the development of neurodegenerative diseases. Greilberger et al (6) compared the redox state of the blood between healthy individuals and patients with neurodegenerative diseases. Compared with healthy controls, there were significant elevations in malondialdehyde, protein carbonylation and oxidized albumin levels in patients with neurodegenerative diseases, indicating that there is an association between oxidative stress and the development of neurodegenerative diseases (6). Furthermore, 4-hydroxynonenal-modified proteins have been detected in patients with mild cognitive impairments and throughout the course of Alzheimer's disease (7). In addition, oxidative stress may serve an important role in dopaminergic neurotoxicity (8) and dopamine metabolism-induced alterations in redox status may increase the risk of the onset or progression of Parkinson's disease (9).

The fruits of Lycium barbarum L. (family Solanaceae), more commonly known as Goji berry or wolfberry, have been used in traditional Chinese herbal medicine for thousands of years. Lycium barbarum polysaccharide (LBP) is the major active component found in the fruits. It has been demonstrated that LBP possesses various biological activities, including antioxidant, anti-cancer, anti-inflammatory, anti-aging and immune-regulatory activities (10-15). In particular, it has recently been demonstrated that LBP exhibits potent neuroprotective effects in vivo and in vitro (16-18). 
Bie et al (16) reported that LBP improved bipolar pulse current-induced microglia cell injury via modulation of autophagy. Furthermore, LBP improved traumatic cognition by reversing the imbalance of apoptosis/regeneration in hippocampal neurons following stress (17). Wang et al (18) determined that LBP prevented focal cerebral ischemic injury by inhibiting neuronal apoptosis in mice. However, the mechanism of the neuroprotective effect of LBP remains unclear.

The present study aimed to investigate the mechanism of the LBP-exerted protective effect against oxidative stress-induced neurotoxicity in vivo and in vitro. The results demonstrated that LBP exhibited neuroprotective activity via activation of nuclear factor erythroid 2-related factor 2 (Nrf) 2/heme oxygenase (HO)-1 signaling.

\section{Materials and methods}

Reagents. $\beta$-actin (sc-130300) and Nrf2 (sc-722) antibodies were purchased from Santa Cruz Biotechnology, Inc. (Dallas, TX, USA) and the HO-1 (RT1270) antibody was purchased from Epitomics; Abcam (Cambridge, MA, USA). MTT and $\mathrm{CoCl}_{2}$ were purchased from Sigma-Aldrich; Merck KGaA (Darmstadt, Germany). Lipofectamine ${ }^{\circledR} 2000$, the intracellular superoxide probe dihydroethidium (DHE) and Rhodamine (Rho) 123 were all purchased from Invitrogen; Thermo Fisher Scientific, Inc. (Waltham, MA, USA).

Cell culture and treatment. PC12 cells were purchased from the American Type Culture Collection (Manassas, VA, USA). Cells were cultured in Dulbecco's modified Eagle's medium (DMEM) (Gibco; Thermo Fisher Scientific, Inc.) supplemented with $10 \%$ fetal calf serum (Gibco; Thermo Fisher Scientific, Inc.), $100 \mu \mathrm{g} / \mathrm{ml}$ penicillin and $100 \mathrm{U} / \mathrm{ml}$ streptomycin at $37^{\circ} \mathrm{C}$ with $5 \% \mathrm{CO}_{2}$ in a humidified incubator. Throughout the experimental treatments, cells were exposed to $\mathrm{H}_{2} \mathrm{O}_{2}$ in the presence or absence of LBP in serum-free DMEM.

Cell transfection. To test the role of Nrf2 in LBP-exerted protective effect, $\mathrm{PC} 12$ cells were transfected with small interfering RNA (siRNA) of Nrf2 and control siRNA before the treatment of $\mathrm{H}_{2} \mathrm{O}_{2}$ and LBP. Nrf2 siRNA and control siRNA were purchased from Santa Cruz Biotechnology (sc-156128). Transfection was conducted using Lipofectamine 2000 reagent (Invitrogen; Thermo Fisher Scientific, Inc.) according to the manufacturer's protocol.

Determination of cell viability. Cells were seeded in a 96-well plate at a density of $1 \times 10^{4}$ cells/well. Cells in the exponential phase were exposed to $0,50,100,200$ or $400 \mu \mathrm{M} \mathrm{H}_{2} \mathrm{O}_{2}$ and/or $125,250,500,800$ or $1,000 \mu \mathrm{g} / \mathrm{ml}$ LBP for $24 \mathrm{~h}$. In some experiments, cells were transfected with Nrf2 siRNA of Nrf2 and control siRNA for $48 \mathrm{~h}$ prior to treatment. Cells were then exposed to $\mathrm{H}_{2} \mathrm{O}_{2}$ and/or LBP in the presence of zinc protoporphyrin IX (ZnPP) (10 $\mu \mathrm{M}$; Sigma-Aldrich; Merck KGaA), an inhibitor of HO-1. Following the experiments, cell viability was determined by MTT assay. The purple formazan was dissolved in dimethyl sulfoxide. Absorbance was measured at $550 \mathrm{~nm}$ and the results are presented as folds of the control cells without $\mathrm{H}_{2} \mathrm{O}_{2}$ treatment.
Determination of apoptosis. Cells $\left(5 \times 10^{5}\right)$ were seeded in culture dishes and treated with $400 \mu \mathrm{M} \mathrm{H}_{2} \mathrm{O}_{2}$ in the presence or absence of 125,250 or $500 \mu \mathrm{g} / \mathrm{ml} \mathrm{LBP}$ with or without ZnPP for $24 \mathrm{~h}$. In some experiments, cells were transfected with siRNAs for $48 \mathrm{~h}$ prior to treatment. Following treatment, cells were fixed with $4 \%$ formaldehyde for $15 \mathrm{~min}$ at room temperature. Following washing with PBS, the cells were covered with proteinase K solution for 15-20 min. After another PBS wash, the cells were covered with the TUNEL reaction mixture (Roche Applied Science, Penzberg, Germany) and incubated for $1 \mathrm{~h}$ in the dark. DAPI counterstaining (10 $\mathrm{min}$ at room temperature) was followed by a final PBS wash, and tissue sections were then examined and photographed using confocal microscopy. The average number of fluorescent dots in three images from each treatment group was calculated. Results were expressed as folds of the control.

Determination of caspase activity. Activities of caspase 3 and 9 were determined using a Caspase 3 Activity Assay kit (C1115) and Caspase 9 Activity Assay kit (C1157; Beyotime Institute of Biotechnology, Haimen, China) according to the manufacturer's protocol.

Determination of ROS levels. ROS levels were measured using oxidation-sensitive probes. Cells were incubated with $10 \mu \mathrm{M}$ DHE for $20 \mathrm{~min}$ at $37^{\circ} \mathrm{C}$ in the dark and were subsequently observed under a confocal fluorescence microscope. In addition, cells were also incubated with $10 \mu \mathrm{M}$ 2,7-Dichlorodihydrofluorescein diacetate (DCFH-DA, an intracellular hydrogen peroxide probe) (Sigma-Aldrich; Merck $\mathrm{KGaA}$ ) for $30 \mathrm{~min}$ at $37^{\circ} \mathrm{C}$ in the dark. Subsequently, cells were washed twice with PBS and analyzed using a flow cytometer (BD Accuri ${ }^{\mathrm{TM}}$ C6, BD Biosciences, Franklin Lakes, NJ, USA) and BD Accuri C6 software (BD Biosciences). The results were expressed as folds of the control.

Mitochondrial membrane potential. After the treatment of $\mathrm{H}_{2} \mathrm{O}_{2}$ and LBP, cells were stained with Rhodamine 123 $(10 \mu \mathrm{M})$ for $20 \mathrm{~min}$ at $37^{\circ} \mathrm{C}$ in the dark. Then the fluorescence was observed under a confocal fluorescence microscope.

Determination of transcription activity. A reporter gene assay was conducted to evaluate the transcriptional activity of Nrf2. PC12 cells $\left(5 \times 10^{4}\right)$ were seeded into 12 -well plates. Subsequently, cells were transfected with $0.2 \mu \mathrm{g}$ antioxidant response element (ARE)-Luc constructs (Beyotime Institute of Biotechnology, Haimen, China) using Lipofectamine 2000 , according to the manufacturer's protocol. pRL-null plasmid (50 ng) encoding Renilla luciferase was included in all samples to ensure transfection efficiency. A total of $48 \mathrm{~h}$ after transfection, cells were treated with $400 \mu \mathrm{M} \mathrm{H}_{2} \mathrm{O}_{2}$ in the presence or absence of 125,250 or $500 \mu \mathrm{g} / \mathrm{ml} \mathrm{LBP}$ for $24 \mathrm{~h}$. Firefly and Renilla luciferase activities were detected sequentially using the Dual-Glo ${ }^{\mathrm{TM}}$ Luciferase assay system (Promega Corporation, Madison, WI, USA).

Chromatin immunoprecipitation (ChIP) assay. Cells were treated with $400 \mu \mathrm{M} \mathrm{H}_{2} \mathrm{O}_{2}$ in the presence or absence of $500 \mu \mathrm{g} / \mathrm{ml} \mathrm{LBP}$ for $24 \mathrm{~h}$. Subsequently, a ChIP assay was conducted to examine the binding of Nrf2 in HO-1 promoters. 
Following the experiment, cells were washed, fixed in $1 \%$ formaldehyde for $10 \mathrm{~min}$ at room temperature and sonicated (on $3 \mathrm{sec}$ and off $10 \mathrm{sec}$ for 10 times at room temperature) to shear chromatin. Nrf2 antibody (dilution 1:50) was added to the cleared lysate and binding was allowed to proceed at $4^{\circ} \mathrm{C}$ overnight. The complex was eluted and the released DNA was amplified by reverse transcription-quantitative polymerase chain reaction (RT-qPCR) as stated below.

$R N A$ isolation and $R T-q P C R$. Total RNA was isolated from cells and tissues using Total RNA Isolation kit (Tiangen Biotech, Co., Ltd., Beijing, China) according to the manufacturer's instructions. A total of $0.5 \mu \mathrm{g}$ RNA was reverse transcribed using the First Strand cDNA synthesis kit (Takara Bio, Inc., Otsu, Japan). qPCR was performed to precisely quantify Nrf2 and HO-1 using SYBR Green reagent (Takara Bio, Inc.). Amplification was performed with an initial step at $94^{\circ} \mathrm{C}$ for $5 \mathrm{~min}$, followed by 40 cycles of denaturation at $94^{\circ} \mathrm{C}$ for $30 \mathrm{sec}$, annealing at $63^{\circ} \mathrm{C}$ for $30 \mathrm{sec}$ and extension at $72^{\circ} \mathrm{C}$ for $10 \mathrm{sec}$. The $2^{-\triangle \Delta \mathrm{Cq}}$ method was used to determine gene expression compared with the endogenous controls (GAPDH) (19). The primers for qPCR analysis were as follows: Nrf2, forward, 5'-ATTGCCTGTAAGTCCTGGTCA-3', reverse, 5'-ACTGCT CTTTGGACATCATTTCG-3'; HO-1, forward, 5'-TTCCTG GACTGATCCCAATTCTG-3', reverse, 5'-CTTGGAAGC CACAGAAATGCAG-3'; GAPDH, forward, 5'-GCACCG TCAAGGCTGAGAAC-3', reverse, 5'-TGGTGAAGACGC CAGTGGA-3'.

Protein extraction and western blotting. Following treatment, cells were lysed and cytoplasmic and nuclear proteins were extracted using NE-PER Nuclear and Cytoplasmic Extraction reagents (Thermo Fisher Scientific, Inc.). Protein concentration was determined using the BCA assay. Lysates containing $20 \mu \mathrm{g}$ protein were boiled at $100^{\circ} \mathrm{C}$ for $5 \mathrm{~min}$, separated on a $10 \%$ sodium dodecyl sulfate-polyacrylamide gel and transferred to PVDF membranes. Membranes were blocked in $8 \%$ skim milk in TBS buffer for $30 \mathrm{~min}$ at $37^{\circ} \mathrm{C}$. Subsequently, membranes were incubated with the appropriate primary antibodies (Nrf2, HO-1 and $\beta$-actin, dilution: 1:500) overnight at $4^{\circ} \mathrm{C}$ and washed four times with TBST for 10 min each time. Following washing, membranes were further incubated with horseradish peroxidase (HRP)-conjugated secondary antibodies (goat anti-rabbit antibody, dilution: 1:5,000) (32260; Invitrogen, Thermo Fisher Scientific, Inc.) for $30 \mathrm{~min}$ at $37^{\circ} \mathrm{C}$ and then washed another four times. Membranes were visualized using Immobilon Western Chemiluminescent HRP Substrate (Merck KGaA). Bands were captured using a VersaDoc image analysis system (Bio-Rad Laboratories, Inc., Hercules, CA, USA) and quantified with Quantity One software v.4.62 (Bio-Rad Laboratories, Inc.).

Animal treatment. All animal care was conducted in accordance with the guidelines for the Care and Use of Laboratory Animals (15) and the principles presented by School of Medicine, Xi'an Jiaotong University (Shaanxi, China). The present study was approved by the Ethics Committee of Xi'an High-Tech Hospital. All efforts were made to reduce suffering and the number of animals used. A total of 64 adult male Wistar rats weighing 250-300 g (8-10 weeks old) were obtained from the Animal Centre of the School of Medicine, Xi'an Jiaotong University. Rats were housed in a controlled environment, with a 12-12 h light/dark cycle, controlled humidity (50\%) and temperature $\left(24^{\circ} \mathrm{C}\right)$, and had free access to standard laboratory rat food and water.

Rats were randomly divided into four groups with 16 animals in each group: Control group, $\mathrm{CoCl}_{2}$ group, $\mathrm{CoCl}_{2}+\mathrm{LBP}$ group and $\mathrm{CoCl}_{2}+\mathrm{LBP}+\mathrm{ZnPP}$ group. Surgery was conducted as previously reported (20-22). Control group: Rats received saline solution. $\mathrm{CoCl}_{2}$ group: Rats received $50 \mathrm{mM} \mathrm{CoCl}$. $\mathrm{CoCl}_{2}+\mathrm{LBP}$ group: Rats received $50 \mathrm{mM} \mathrm{CoCl}$ and $\mathrm{LBP}$ $\left(100 \mathrm{mg} / \mathrm{kg}\right.$ ) injection. $\mathrm{CoCl}_{2}+\mathrm{LBP}+\mathrm{ZnPP}$ group: Rats received $50 \mathrm{mM} \mathrm{CoCl}_{2}, \mathrm{LBP}(100 \mathrm{mg} / \mathrm{kg})$ injection and $\mathrm{ZnPP}(10 \mathrm{mg} / \mathrm{kg})$ injection. Briefly, rats were placed in a stereotaxic apparatus and received a unilateral lesion by drilling a small hole on the skull in the frontoparietal cortex at Bregma- $1.30 \mathrm{~mm}$. Subsequently, a sterile solution of $\mathrm{CoCl}_{2}(50 \mathrm{mM})$ or an equivalent volume of saline solution was injected in the right hemisphere, $1 \mathrm{~mm}$ below the pia level in the cerebral cortex (layers 3-4) with a Hamilton syringe. The $\mathrm{CoCl}_{2}$ solution was adjusted to reach a physiological osmolarity of $310 \mathrm{mOsm} / \mathrm{kg}$. Following the injection, the lesion site was repaired using the temporal muscle and the attached fascia. During the surgery period, the body temperature of the animals was maintained using a heating pad. Surgical procedures were performed under anesthesia induced with $8 \%(\mathrm{v} / \mathrm{v})$ sevofluorane inhalation (the dose was selected by preliminary experiments). Concurrent preemptive analgesia was used and a precision vaporiser was used to perform anaesthesia and each animal was put in a separate cage for recovery. Following 2 days recovery, rats in $\mathrm{CoCl}_{2}+\mathrm{LBP}$ group and $\mathrm{CoCl}_{2}+\mathrm{LBP}+\mathrm{ZnPP}$ group received intraperitoneal $\mathrm{LBP}(100 \mathrm{mg} / \mathrm{kg})$ and rats in $\mathrm{CoCl}_{2}+\mathrm{LBP}+\mathrm{ZnPP}$ group received $\mathrm{ZnPP}(10 \mathrm{mg} / \mathrm{kg})$ injection once a day for 10 days. After the treatment, 8 rats in each group were sacrificed using intraperitoneal injection of an overdose of sodium pentobarbital (200 mg/kg; Merck KGaA). Frozen brain sections $(5 \mu \mathrm{m})$ were cut and then fixed in $4 \%$ formaldehyde overnight at room temperature for the determination of apoptosis.

Morris water maze (MWM) test. Immediately after the treatment, an MWM test was conducted to evaluate spatial learning and memory abilities, as previously reported $(22,23)$. The maze was a black circular pool $(120 \mathrm{~cm}$ in diameter and $45 \mathrm{~cm}$ high) divided into four quadrants, with a depth of $30 \mathrm{~cm}$ and filled with clear tap water at a temperature of $27 \pm 0.5^{\circ} \mathrm{C}$. A black platform $10 \mathrm{~cm}$ in diameter was located $2 \mathrm{~cm}$ below the surface of the water approximately in the middle of one of the four quadrants. Each rat's path in the pool was recorded with Videomex Water Maze Monitoring Software BL-420 (Chengdu Taimeng Technology Co., Ltd., Chengdu, China) and analyzed off-line. Experiments were conducted in a soundproof room and the light source and surrounding environment remained unaltered between each experiment.

During the first 5 days, rats underwent four swimming trials per day, with each trial period limited to $60 \mathrm{sec}$. In the trials, the time taken to reach the platform, the swimming distance and the speed were recorded. If the rat did not find the platform within the set time, the computer would stop tracking and record the time as $60 \mathrm{sec}$. If the platform was found within the $60 \mathrm{sec}$, the rat would be allowed to remain 
A

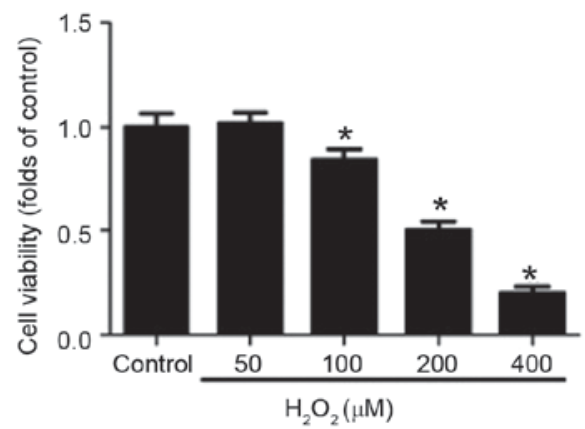

C

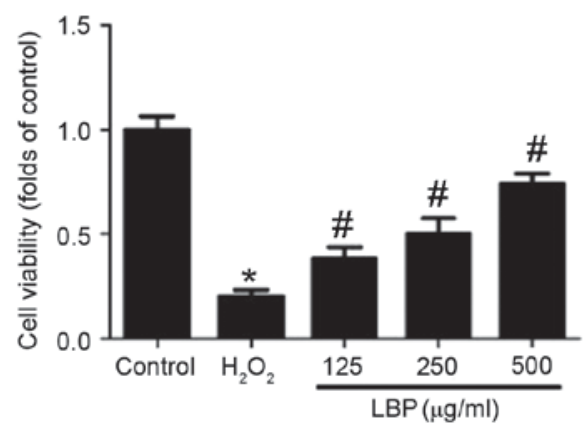

B

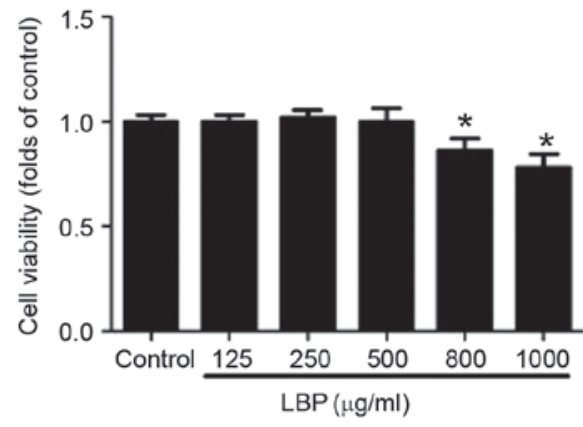

$\mathrm{D}$

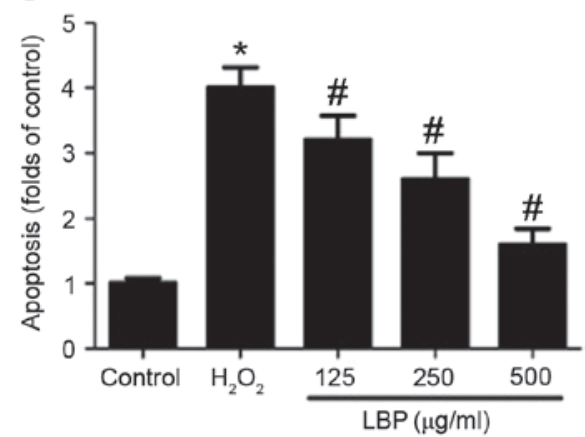

Figure 1. Effect of LBP on $\mathrm{H}_{2} \mathrm{O}_{2}$-induced apoptosis. PC12 cells were incubated with (A) 0, 50, 100, 200 or $400 \mu \mathrm{M} \mathrm{H}_{2} \mathrm{O}_{2}$ or (B) $0,125,250,500,800$ or $1,000 \mu \mathrm{g} / \mathrm{ml} \mathrm{LBP}$ for $24 \mathrm{~h}$. Cell viability was determined by MTT assay and the results are presented as folds of control. (C) PC12 cells were incubated with $400 \mu \mathrm{M} \mathrm{H}_{2} \mathrm{O}_{2}$ in the presence or absence of 125,250 or $500 \mu \mathrm{g} / \mathrm{ml} \mathrm{LBP}$ for $24 \mathrm{~h}$. Cell viability was determined by MTT assay and the results are presented as folds of control. (D) Apoptosis was determined by TUNEL staining and the results are presented as folds of control. ${ }^{*} \mathrm{P}<0.05$ vs. control; ${ }^{*} \mathrm{P}<0.05$ vs. $\mathrm{H}_{2} \mathrm{O}_{2}$ treatment. LBP, Lycium barbarum polysaccharide.

on the platform for $10 \mathrm{sec}$. Otherwise, the rat would be guided to the platform to remain for $10 \mathrm{sec}$. On day 6 , a probe trial test was conducted. The platform was removed on day 6 for a $60 \mathrm{sec}$ exploration test to record the crossing index to the previous platform site.

Statistical analysis. Statistical analysis was performed using GraphPad Software 5.0 (GraphPad Software, Inc., La Jolla, CA, USA). The results are presented as the mean \pm standard error of the mean. The statistical significance of differences between groups was analyzed via one-way analysis of variance followed by a Dunnett's t-test for multiple comparisons. $\mathrm{P}<0.05$ was considered to indicate a significant difference.

\section{Results}

LBP inhibits $\mathrm{H}_{2} \mathrm{O}_{2}$-induced mitochondrial apoptosis in PC12 cells. PC12 cells were incubated with $0,50,100,200$ or $400 \mu \mathrm{M}$ $\mathrm{H}_{2} \mathrm{O}_{2}$ for $24 \mathrm{~h}$ and the results indicated that $100-400 \mu \mathrm{M} \mathrm{H}_{2} \mathrm{O}_{2}$ significantly decreased cell viability in a concentration-dependent manner ( $\mathrm{P}<0.05$; Fig. 1A). Treatment with $400 \mu \mathrm{M} \mathrm{H}_{2} \mathrm{O}_{2}$ decreased cell viability to $20 \%$ of the control; thus $400 \mu \mathrm{M}$ $\mathrm{H}_{2} \mathrm{O}_{2}$ was used to induce oxidative injury in PC12 cells in subsequent experiments. PC12 cells were incubated with 0 , $125,250,500,800$ or $1,000 \mu \mathrm{g} / \mathrm{ml} \mathrm{LBP}$ for $24 \mathrm{~h}$ and the results indicated that LBP significantly decreased cell viability in a concentration-dependent manner at concentrations $>500 \mu \mathrm{g} / \mathrm{ml}$ ( $\mathrm{P}<0.05$; Fig. 1B). Thus, a concentration of $125-500 \mu \mathrm{g} / \mathrm{ml} \mathrm{LBP}$ was selected to examine the effect of LBP on neurotoxicity in PC12 cells in subsequent experiments.
LBP dose-dependently reversed the decrease in cell viability induced by $\mathrm{H}_{2} \mathrm{O}_{2}(\mathrm{P}<0.05$; Fig. 1C). Furthermore, LBP treatment decreased TUNEL-positive cell numbers in $\mathrm{H}_{2} \mathrm{O}_{2}$-treated cells in a concentration-dependent manner ( $\mathrm{P}<0.05$; Fig. 1D). $\mathrm{H}_{2} \mathrm{O}_{2}$-induced increases in caspase-3 and -9 activity were significantly reversed by LBP in a concentration-dependent manner $(\mathrm{P}<0.05$; Fig. 2A and B). Mitochondrial membrane potential was determined using Rho 123 staining and fluorescence was observed using a confocal microscope. The results demonstrated that LBP markedly inhibited the $\mathrm{H}_{2} \mathrm{O}_{2}$-induced decrease in Rho 123 fluorescence, indicating a reversal in the decrease of the mitochondrial membrane potential (Fig. 2C). ROS levels were determined by DHE and DCFH-DA staining. The $\mathrm{H}_{2} \mathrm{O}_{2}$-induced increase in DHE staining was markedly inhibited by LBP (Fig. 2D). Furthermore, LBP significantly suppressed the $\mathrm{H}_{2} \mathrm{O}_{2}$-induced increase of DCFH-DA-positive cell numbers in a concentration dependent manner $(\mathrm{P}<0.05$; Fig. $2 \mathrm{E})$. These results indicate that LBP concentration-dependently inhibits $\mathrm{H}_{2} \mathrm{O}_{2}$-induced mitochondrial apoptosis.

LBP inhibits the $\mathrm{H}_{2} \mathrm{O}_{2}$-induced decrease of $\mathrm{Nrf} 2 / \mathrm{HO}-1$ signaling in PC12 cells. The potential mechanism responsible for the LBP-induced protective effects against $\mathrm{H}_{2} \mathrm{O}_{2}$-induced neurotoxicity in PC12 cells was subsequently evaluated. Levels of Nrf2 and HO-1 mRNA and protein were measured. The results demonstrated that the $\mathrm{H}_{2} \mathrm{O}_{2}$-induced decreases in Nrf2 and HO-1 mRNA expression were significantly reversed by LBP in a concentration-dependent manner $(\mathrm{P}<0.05$; Fig. $3 \mathrm{~A}$ and $\mathrm{B})$. LBP also markedly reversed the decrease in Nrf2 and HO-1 protein expression induced by $\mathrm{H}_{2} \mathrm{O}_{2}$ (Fig. 3C). 
A
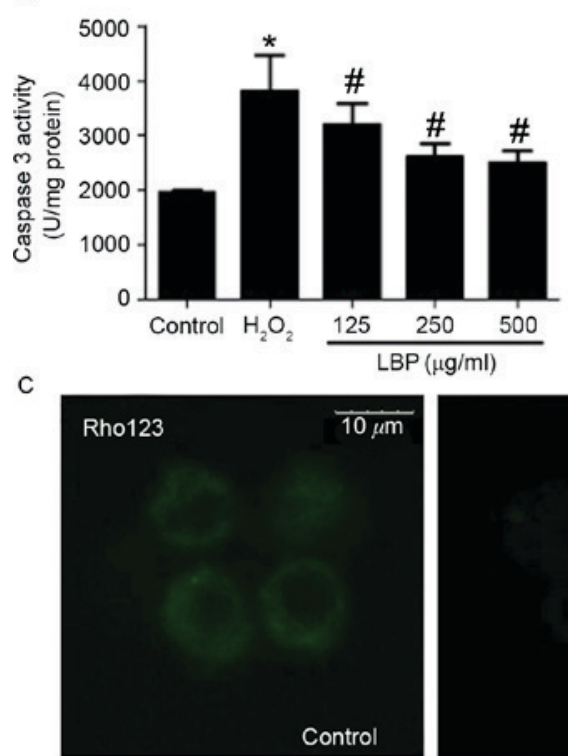

D

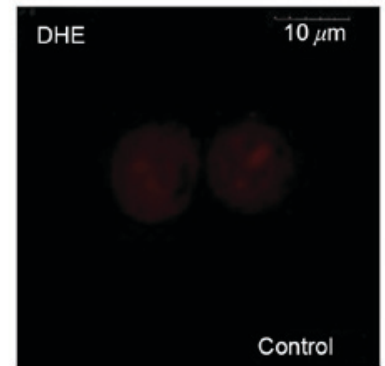

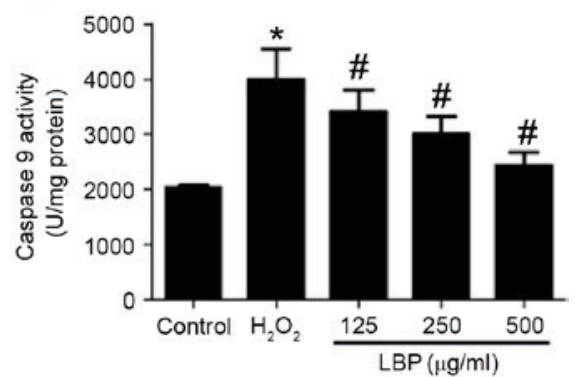
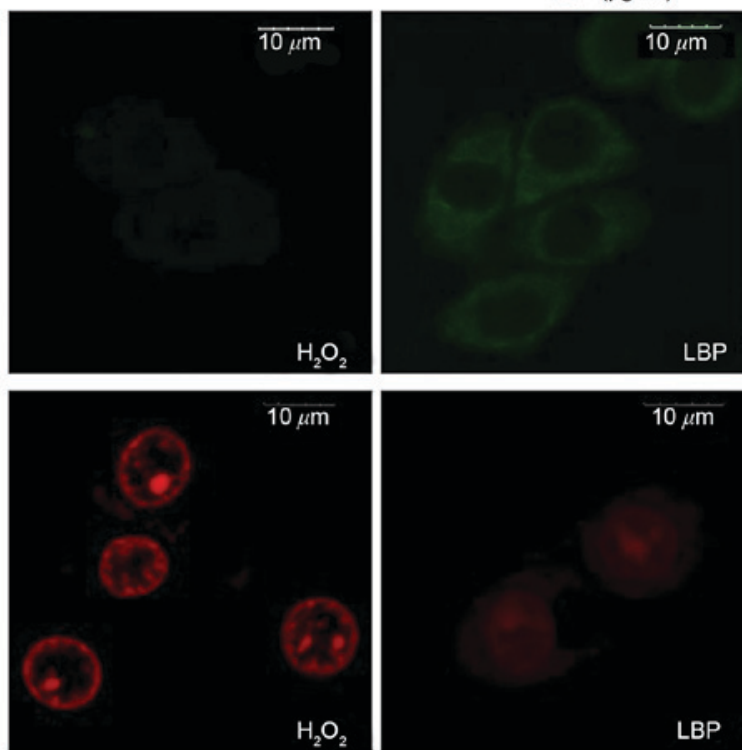

E

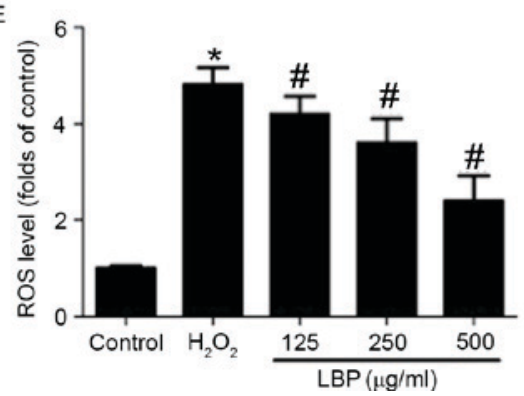

Figure 2. Effect of LBP on the $\mathrm{H}_{2} \mathrm{O}_{2}$-induced mitochondrial apoptotic pathway. PC12 cells were incubated with $400 \mu \mathrm{M} \mathrm{H}_{2} \mathrm{O}_{2}$ in the presence or absence of 125, 250 or $500 \mu \mathrm{g} / \mathrm{ml} \mathrm{LBP}$ for $24 \mathrm{~h}$. (A) Caspase-3 and (B) -9 activities were measured using commercial kits and expressed as U/mg protein. (C) PC12 cells were incubated with $400 \mu \mathrm{M} \mathrm{H}_{2} \mathrm{O}_{2}$ in the presence or absence of $500 \mu \mathrm{g} / \mathrm{ml} \mathrm{LBP}$ for $24 \mathrm{~h}$. Mitochondrial membrane potential was detected using Rho123 staining. Representative images are presented. ROS levels were determined by DHE and DCFH-DA staining. (D) For DHE staining, a representative image is presented. (E) For DCFH-DA staining, fluorescence was analyzed by flow cytometry and the results are presented as folds of control. * $<0.05$ vs. control; ${ }^{\#} \mathrm{P}<0.05$ vs. $\mathrm{H}_{2} \mathrm{O}_{2}$ treatment. LBP, Lycium barbarum polysaccharide; ROS, reactive oxygen species; DCFH-DA, 7-Dichlorodihydrofluorescein-diacetate; DHE, dihydroethidium; Rho123, Rhodamine 123.

ARE-luciferase activity was significantly reduced by $\mathrm{H}_{2} \mathrm{O}_{2}$ $(\mathrm{P}<0.05)$, however this reduction was reversed by LBP $(\mathrm{P}<0.05$; Fig. 3D). The results of the ChIP assay identified that LBP significantly reversed the $\mathrm{H}_{2} \mathrm{O}_{2}$-induced decrease of Nrf2 binding to the promoters of HO-1 ( $\mathrm{P}<0.05$; Fig. $3 \mathrm{E}$ ). Cells were transfected with siNrf2 and then exposed to $\mathrm{H}_{2} \mathrm{O}_{2}$ in the presence or absence of LBP. The results determined that the LBP-induced increase of HO-1 expression in $\mathrm{H}_{2} \mathrm{O}_{2}$-treated cells was significantly inhibited by Nrf2 silencing induced by siNrf2 ( $\mathrm{P}<0.05$; Fig. 3F). These results suggest that Nrf2/HO-1 signaling is involved in the LBP-induced protective effects against neurotoxicity induced by $\mathrm{H}_{2} \mathrm{O}_{2}$ and indicate that LBP inhibits the $\mathrm{H}_{2} \mathrm{O}_{2}$-induced decrease of Nrf2/HO-1 signaling in PC12 cells. To determine whether the upregulation of
Nrf2/HO-1 signaling is involved in the protective effect of LBP, cells were transfected with siNrf2, then exposed to $\mathrm{H}_{2} \mathrm{O}_{2}$ in the presence or absence of LBP with or without $\mathrm{ZnPP}$, an inhibitor of HO-1. It was demonstrated that in $\mathrm{H}_{2} \mathrm{O}_{2}$-treated cells, the LBP-induced decrease in ROS levels (Fig. 4A), increase in cell viability (Fig. 4B) and decrease in cell apoptosis (Fig. 4C) was significantly reversed by siNrf 2 and $\mathrm{ZnPP}$ (all $\mathrm{P}<0.05$ ). These results indicate that $\mathrm{Nrf} 2 / \mathrm{HO}-1$ signaling is involved in the LBP-induced protective effects against neurotoxicity induced by $\mathrm{H}_{2} \mathrm{O}_{2}$ in $\mathrm{PC} 12$ cells.

Neuroprotective effect of $L B P$ on $\mathrm{CoCl}_{2}$ in vivo. Subsequently, the neuroprotective effect of LBP against $\mathrm{CoCl}_{2}$ in vivo was evaluated. Rats were injected with $\mathrm{CoCl}_{2}$ to construct an 

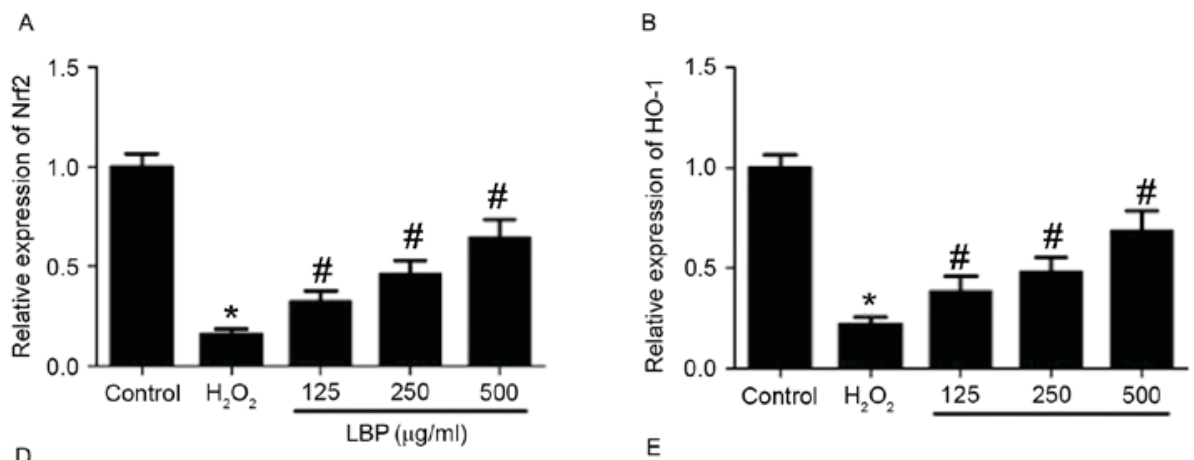

C
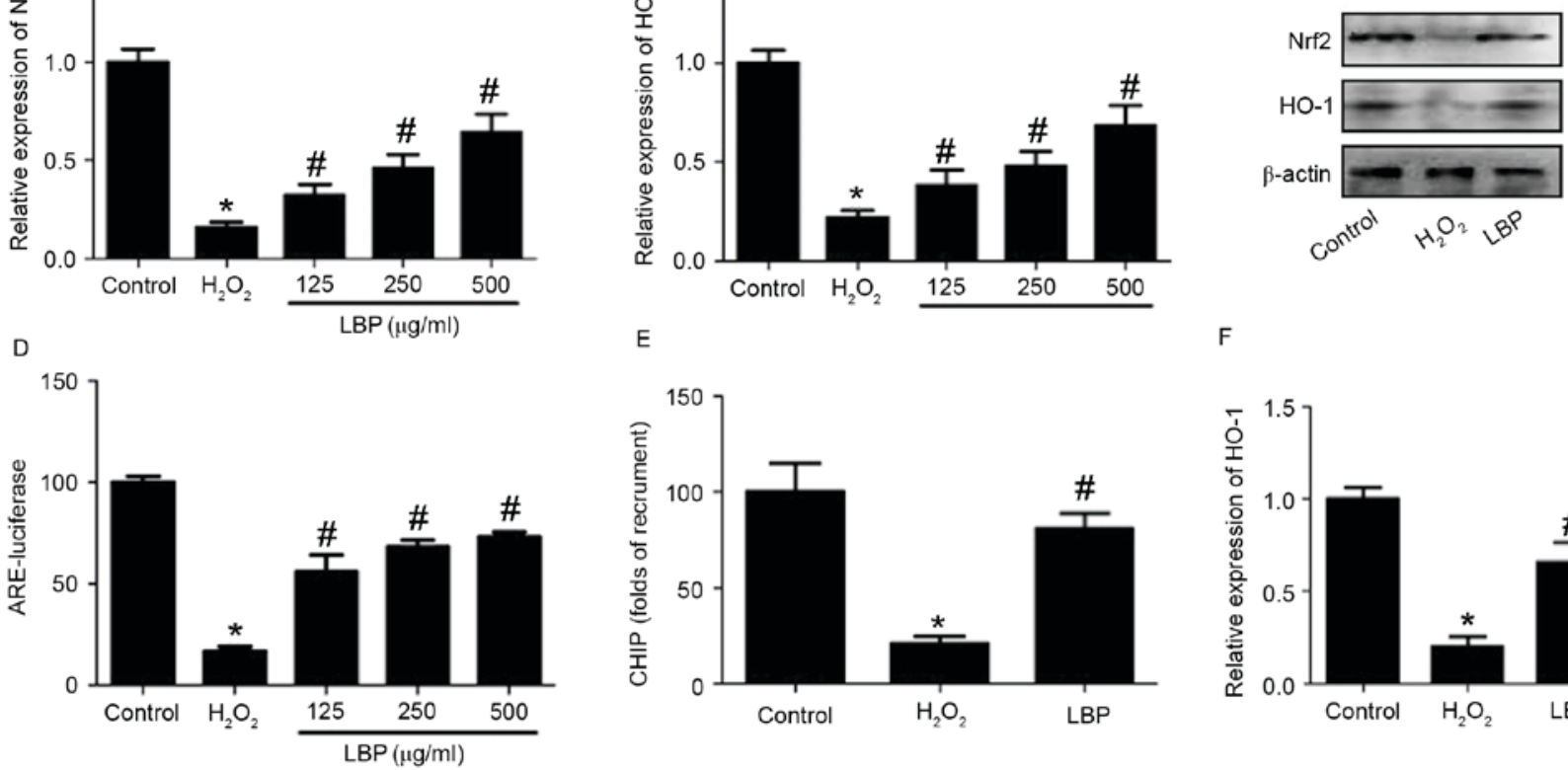

Figure 3. Effect of LBP on the $\mathrm{H}_{2} \mathrm{O}_{2}$-induced decrease of $\mathrm{Nrf} 2 / \mathrm{HO}-1$ signaling. $\mathrm{PC} 12$ cells were incubated with $400 \mu \mathrm{M} \mathrm{H}_{2} \mathrm{O}_{2}$ in the presence or absence of 125,250 or $500 \mu \mathrm{g} / \mathrm{ml}$ LBP for $24 \mathrm{~h}$. (A and B) mRNA and (C) protein expression of Nrf2 and HO-1 was determined by RT-qPCR and western blotting, respectively. (D) Transcriptional activity of Nrf2 was detected by ARE-luciferase activity. (E) Direct regulation of HO-1 by Nrf2 was determined using

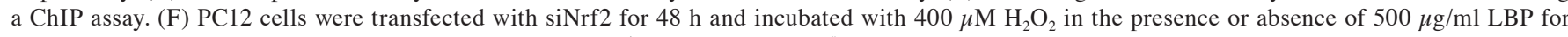
24 h. HO-1 mRNA expression was determined by RT-qPCR. " $\mathrm{P}<0.05$ vs. control; ${ }^{*} \mathrm{P}<0.05$ vs. $\mathrm{H}_{2} \mathrm{O}_{2}$ treatment; ${ }^{\wedge} \mathrm{P}<0.05$ vs. LBP treatment. RT-qPCR, reverse transcription-quantitative polymerase chain reaction; LBP, Lycium barbarum polysaccharide; Nrf2, nuclear factor erythroid 2-related factor 2; HO-1, heme oxygenase-1; ChIP, Chromatin immunoprecipitation.

A

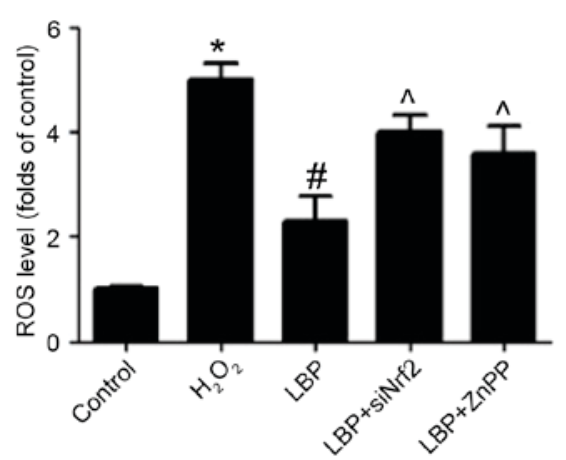

B

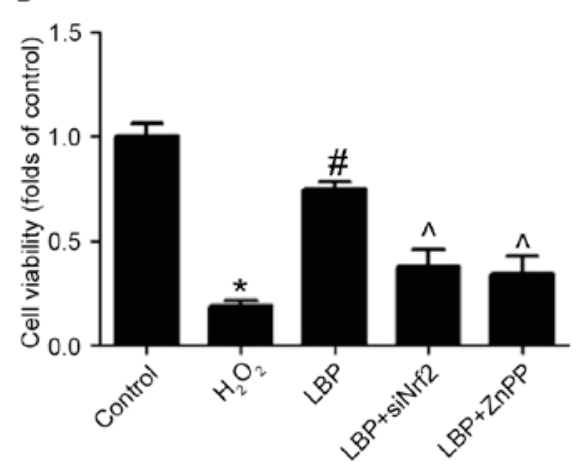

C

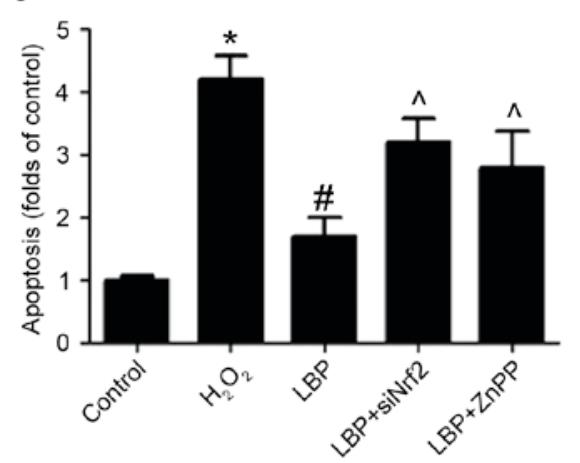

Figure 4. Role of Nrf2/HO-1 signaling in LBP-induced effects on $\mathrm{H}_{2} \mathrm{O}_{2}$-treated cells. PC12 cells were transfected with siNrf2 for 48 h and subsequently incubated with $400 \mu \mathrm{M} \mathrm{H}_{2} \mathrm{O}_{2}$ in the presence or absence of $500 \mu \mathrm{g} / \mathrm{ml} \mathrm{LBP}$ with or without ZnPP for $24 \mathrm{~h}$. (A) ROS levels were determined by staining with DCFH-DA. (B) Cell viability was determined by an MTT assay and the results are presented as folds of the control. (C) Apoptosis was determined by TUNEL staining and the results are presented as folds of the control. ${ }^{*} \mathrm{P}<0.05$ vs. control; ${ }^{*} \mathrm{P}<0.05$ vs. $\mathrm{H}_{2} \mathrm{O}_{2}$ treatment; ${ }^{\wedge} \mathrm{P}<0.05$ vs. LBP treatment. siNrf 2 , small interfering Nrf2 RNA; Nrf2, nuclear factor erythroid 2-related factor 2; HO-1, heme oxygenase-1; ZnPP, zinc protoporphyrin IX; LBP, Lycium barbarum polysaccharide; DCFH-DA, 7-Dichlorodihydrofluorescein-diacetate; ROS, reactive oxygen species.

animal model of neurotoxicity and subsequently received LBP with or without ZnPP. Following treatment, a MWM test was conducted to evaluate the spatial learning and memory abilities of rats. The $\mathrm{CoCl}_{2}$-induced decrease of $\mathrm{Nrf} 2$ expression was significantly inhibited by $\mathrm{LBP}(\mathrm{P}<0.05)$ and $\mathrm{ZnPP}$ did not significantly affect Nrf2 expression in LBP-treated rats (Fig. 5A). LBP reversed the $\mathrm{CoCl}_{2}$-induced decrease of $\mathrm{HO}-1$ expression in rat brains $(\mathrm{P}<0.05$; Fig. $5 \mathrm{~B})$, however this effect was significantly attenuated by $\mathrm{ZnPP}(\mathrm{P}<0.05$; Fig. $5 \mathrm{~B})$. $\mathrm{A} \mathrm{CoCl}_{2}$-induced increase in the number of TUNEL-positive cells was inhibited by $\mathrm{LBP}$, indicating a reduction in apoptosis $(\mathrm{P}<0.05$; Fig. $5 \mathrm{C})$.
However, ZnPP significantly reversed the anti-apoptotic effect of LBP in $\mathrm{CoCl}_{2}$-treated rats $(\mathrm{P}<0.05$; Fig. $5 \mathrm{C})$. During the MWM test, rats in $\mathrm{CoCl}_{2}$ group spent more time finding the platform site, indicated by a significantly lower crossing index compared with control rats $(\mathrm{P}<0.05$; Fig. 5D). Compared with the $\mathrm{CoCl}_{2}$ group, rats in LBP group spent significantly less time finding the platform site $(\mathrm{P}<0.05$; Fig. 5D), however this decrease in time spent to find the platform site induced by LBP was reversed by $\mathrm{ZnPP}$ ( $\mathrm{P}<0.05$; Fig. 5D).

Rats in the $\mathrm{CoCl}_{2}$ group exhibited significantly longer escape latencies on test days 3,4 , and 5 than those in the control 
A

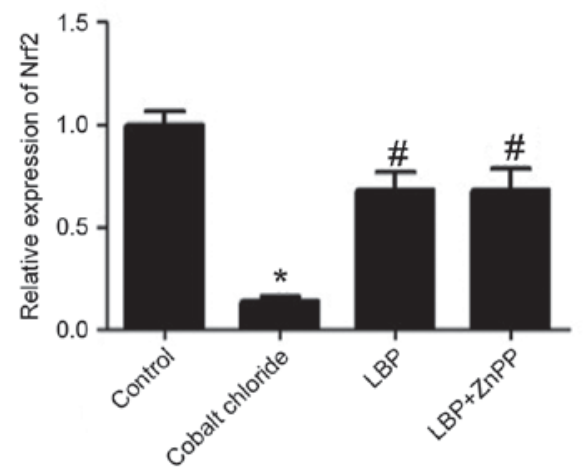

C
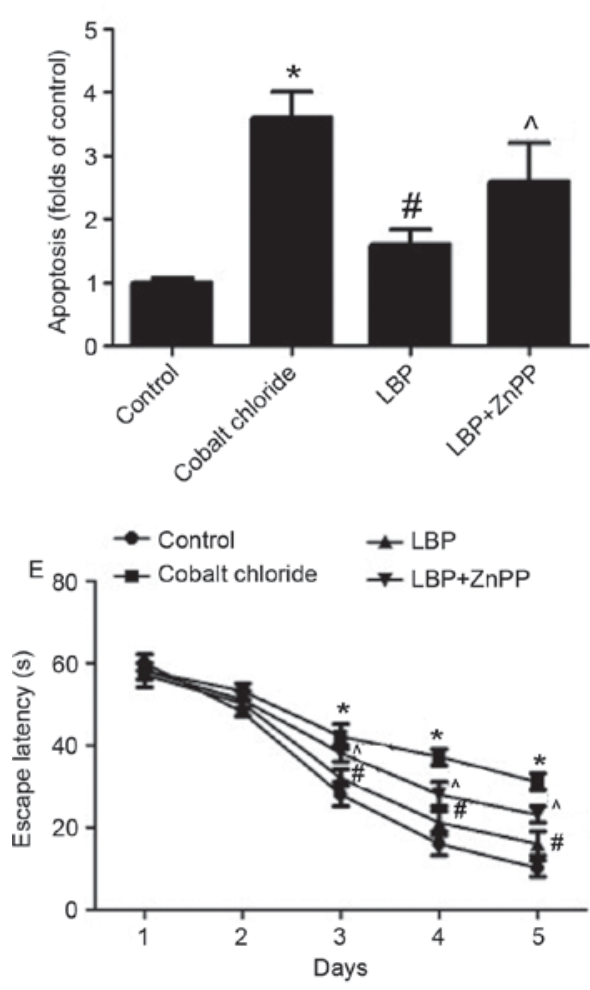

B
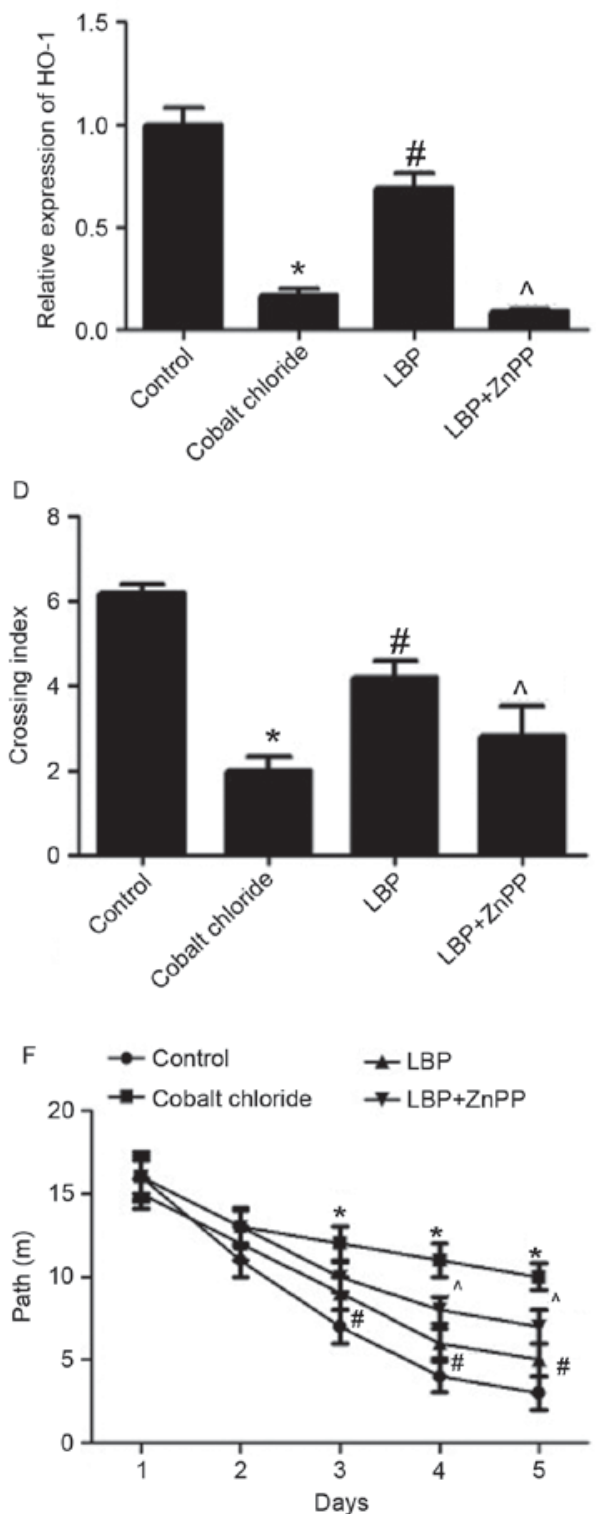

Figure 5. Neuroprotective effect of LBP in rats against $\mathrm{CoCl}_{2}$. Rats were treated with $\mathrm{CoCl}_{2}$ and subsequently received intraperitoneal $\mathrm{LBP}(100 \mathrm{mg} / \mathrm{kg})$ or saline solution with or without $\mathrm{ZnPP}(10 \mathrm{mg} / \mathrm{kg}$ ). The expression of (A) Nrf2 and (B) HO-1 mRNA in brain tissue was determined by reverse transcription-quantitative polymerase chain reaction and the results are presented as folds of the control. (C) Apoptosis was determined by TUNEL staining and results were shown as folds of the control. (D) A MWM test was conducted to evaluate spatial learning and memory abilities of rats. Development of spatial memory for platform location during the probe trials following 5 days training during the MWM test. The crossing index represents the average frequency of swims over the platform site in the target quadrant. (E) Average escape latencies. (F) The distance traveled to find the platform. ${ }^{*} \mathrm{P}<0.05$ vs. control; ${ }^{*} \mathrm{P}<0.05$ vs. $\mathrm{H}_{2} \mathrm{O}_{2}$ treatment; ${ }^{\wedge} \mathrm{P}<0.05$ vs. LBP treatment. Nrf2, nuclear factor erythroid 2-related factor 2; HO-1, heme oxygenase-1; ZnPP, zinc protoporphyrin IX; MWM, morris water maze; LBP, Lycium barbarum polysaccharide.

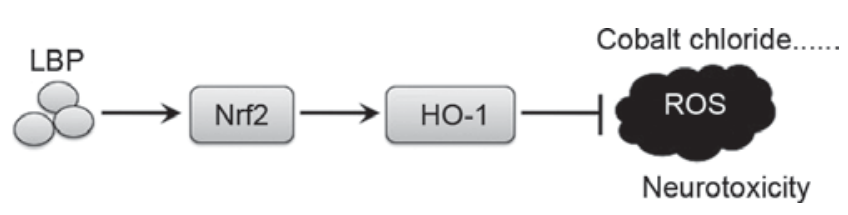

Figure 6. Mechanism of the neuroprotective effect of LBP. LBP, Lycium barbarum polysaccharide; Nrf2, nuclear factor erythroid 2-related factor 2; HO-1, heme oxygenase-1; ROS, reactive oxygen species.

group ( $\mathrm{P}<0.05$; Fig. 5E). Compared with the $\mathrm{CoCl}_{2}$ group, rats in the LBP group had shorter escape latencies on test days 3, 4 , and 5 ( $\mathrm{P}<0.05$; Fig. 5E); however this decrease in escape latencies induced by LBP was reversed by $\mathrm{ZnPP}(\mathrm{P}<0.05$; Fig. 5E). The distance traveled by the rats in each group to find the platform was also gradually reduced by training and the changing patterns of traveling distance induced by LBP and $\mathrm{ZnPP}$ were largely consistent with those for the latency (Fig. 5F). The results demonstrated that LBP may protect against $\mathrm{CoCl}_{2}$-induced neurotoxicity in vivo and that this may involve the regulation of Nrf2/HO-1 signaling (Fig. 6).

\section{Discussion}

There has been a marked increase in the incidence of neurodegenerative diseases including Alzheimer's and Parkinson's 
disease over the last few years $(1,2)$. Previous studies have suggested that oxidative stress serves a crucial role in the development of different types of neurotoxicity (24-26). It has been demonstrated that LBP, the major active component of the Lycium barbarum L. fruit, possesses potent antioxidant activity (27-29), thus the current study examined the potential neuroprotective effect of LBP.

In the current study, in vitro experiments were performed on PC12 cells that had undergone $\mathrm{H}_{2} \mathrm{O}_{2}$-induced neurotoxicity to evaluate the neuroprotective effect of LBP. In addition, $\mathrm{CoCl}_{2}$-mediated hypoxic neurotoxicity was established in rats to examine the neuroprotective effect of LBP in vivo. It was demonstrated that LBP exhibits potent neuroprotective activity, as indicated by the increase in cell viability and decrease in mitochondrial apoptosis in $\mathrm{H}_{2} \mathrm{O}_{2}$-treated $\mathrm{PC} 12$ cells, as well as the decreased apoptosis in rat brain tissue, decreased time to find the platform site, shorter escape latencies and a shorter distance traveled to find the platform in $\mathrm{CoCl}_{2}$-treated rats.

There have been few studies assessing the neuroprotective effects of LBP. It has been demonstrated that LBP improves bipolar pulse current-induced microglia cell injury via modulation of autophagy (16). Furthermore, LBP improved traumatic cognition by reversing the imbalance of apoptosis/regeneration that occurs in hippocampal neurons following the induction of stress (17). Wang et al (18) indicated that LBP prevents focal cerebral ischemic injury by inhibiting neuronal apoptosis in mice. Additionally, Rui et al (30) determined that LBP protects rat primary cultured hippocampal neurons against injury induced by oxygen-glucose deprivation and reperfusion. Li et al (31) demonstrated that LBP reduces neuronal damage, disruption of the blood-retinal barrier and oxidative stress in retinal ischemia/reperfusion injury. Based on the aforementioned results and the results of the current study, LBP may exhibit potent neuroprotective activity.

The antioxidant activities of LBP may serve an important role in the protective effects against neurotoxicity, as reflected by the significant reduction in ROS levels that occurs in $\mathrm{H}_{2} \mathrm{O}_{2}$-treated $\mathrm{PC} 12$ cells. Nrf2 is a key transcription factor that determines redox status by regulating numerous antioxidant enzymes $(32,33)$. HO-1 is an important target gene of Nrf2; HO-1 exhibits antioxidant, anti-apoptotic and anti-inflammatory properties via bilirubin/biliverdin and carbon monoxide, the products of HO-1-catalyzed heme degradation (34). It has been demonstrated that disruption to the Nrf2-ARE pathway contributes to the development of neurotoxicity and neurodegenerative diseases (35-37). Activation of Nrf2/HO-1 signaling may be an important method of protecting against neurotoxicity (38-40). It has been indicated that dietary LBP stimulates the Nrf2/ARE pathway and ameliorates insulin resistance induced by a high-fat diet (41). Furthermore, activation of the Nrf2/HO-1 antioxidant pathway may contribute to the protective effects of LBP in the rodent retina following ischemia-reperfusion-induced damage (42). In the present study, the potential role of Nrf2/HO-1 signaling in the neuroprotective effects of LBP was examined. LBP inhibited the reduction of $\mathrm{Nrf} 2 / \mathrm{HO}-1$ signaling in $\mathrm{H}_{2} \mathrm{O}_{2}$-treated $\mathrm{PC} 12$ cells and $\mathrm{CoCl}_{2}$-treated rats. Nrf2 silencing significantly inhibited the protective effects of LBP in $\mathrm{H}_{2} \mathrm{O}_{2}$-treated $\mathrm{PC} 12$ cells. Furthermore, administration of $\mathrm{ZnPP}$ suppressed the protective effects of $\mathrm{LBP}$ in $\mathrm{H}_{2} \mathrm{O}_{2}$-treated $\mathrm{PC} 12$ cells and $\mathrm{CoCl}_{2}$-treated rats. These results demonstrate that the Nrf2/HO-1 pathway may, at least partly, be responsible for the neuroprotective effects of LBP.

In conclusion, the current study demonstrated that LBP exhibits protective effects against neurotoxicity via upregulation of Nrf2/HO-1 signaling. Enhancement of Nrf2/HO-1 signaling contributed to an improvement of oxidative stress and the amelioration of apoptosis. These data may improve understanding of the neuroprotective activities of LBP.

\section{References}

1. Paulsen JS, Nance M, Kim JI, Carlozzi NE, Panegyres PK, Erwin C, Goh A, McCusker E and Williams JK: A review of quality of life after predictive testing for and earlier identification of neurodegenerative diseases. Prog Neurobiol 110: 2-28, 2013.

2. Gratwicke J, Jahanshahi M and Foltynie T: Parkinson's disease dementia: A neural networks perspective. Brain 138: 1454-1476, 2015.

3. Bhat AH, Dar KB, Anees S, Zargar MA, Masood A, Sofi MA and Ganie SA: Oxidative stress, mitochondrial dysfunction and neurodegenerative diseases; A mechanistic insight. Biomed Pharmacother 74: 101-110, 2015.

4. Zhao Y and Zhao B: Oxidative stress and the pathogenesis of Alzheimer's disease. Oxid Med Cell Longev 2013: 316523, 2013.

5. Wang X and Hai C: Redox modulation of adipocyte differentiation: Hypothesis of 'Redox Chain' and novel insights into intervention of adipogenesis and obesity. Free Radical Bio Med 89: 99-125, 2015.

6. Greilberger J, Koidl C, Greilberger M, Lamprecht M, Schroecksnadel K, Leblhuber F, Fuchs D and Oettl K: Malondialdehyde, carbonyl proteins and albumin-disulphide as useful oxidative markers in mild cognitive impairment and Alzheimer's disease. Free Radic Res 42: 633-638, 2008.

7. Sultana R, Perluigi M and Allan Butterfield D: Lipid peroxidation triggers neurodegeneration: A redox proteomics view into the Alzheimer disease brain. Free Radic Biol Med 62: 157-169, 2013.

8. Blesa J, Trigo-Damas I, Quiroga-Varela A and Jackson-Lewis VR: Oxidative stress and Parkinson's disease. Front Neuroanat 9: 91, 2015.

9. Wong ES, Tan JM, Wang C, Zhang Z, Tay SP, Zaiden N, Ko HS, Dawson VL, Dawson TM and Lim KL: Relative sensitivity of parkin and other cysteine-containing enzymes to stress-induced solubility alterations. J Biol Chem 282: 12310-12318, 2007.

10. Wang HX and Ng TB: Natural products with hypoglycemic, hypotensive, hypocholesterolemic, antiatherosclerotic and antithrombotic activities. Life Sci 65: 2663-2677, 1999.

11. Chen S, Liang L, Wang Y, Diao J, Zhao C, Chen G, He Y, Luo C, Wu X and Zhang Y: Synergistic immunotherapeutic effects of Lycium barbarum polysaccharide and interferon- $\alpha 2 b$ on the murine Renca renal cell carcinoma cell line in vitro and in vivo. Mol Med Rep 12: 6727-6737 2015.

12. Zhao R, Cai Y, Shao X and Ma B: Improving the activity of Lycium barbarum polysaccharide on sub-health mice. Food Funct 6: 2033-2040, 2015

13. Liu Y, Lv J, Yang B, Liu F, Tian Z, Cai Y, Yang D, Ouyang J, Sun F, Shi Y and Xia P: Lycium barbarum polysaccharide attenuates type II collagen-induced arthritis in mice. Int J Biol Macromol 78: 318-323, 2015.

14. Xiao J, Zhu Y, Liu Y, Tipoe GL, Xing F and So KF: Lycium barbarum polysaccharide attenuates alcoholic cellular injury through TXNIP-NLRP3 inflammasome pathway. Int J Biol Macromol 69: 73-78, 2014.

15. Zhao Q, Dong B, Chen J, Zhao B, Wang X, Wang L, Zha S, Wang Y, Zhang J and Wang Y: Effect of drying methods on physicochemical properties and antioxidant activities of wolfberry (Lycium barbarum) polysaccharide. Carbohydr Polym 127: 176-181, 2015.

16. Bie M, Lv Y, Ren C, Xing F, Cui Q, Xiao J and So KF: Lycium barbarum polysaccharide improves bipolar pulse current-induced microglia cell injury through modulating autophagy. Cell Transplant 24: 419-428, 2015.

17. Gao J, Chen C, Liu Y, Li Y, Long Z, Wang H, Zhang Y, Sui J, Wu Y, Liu L and Yang C: Lycium barbarum polysaccharide improves traumatic cognition via reversing imbalance of apoptosis/regeneration in hippocampal neurons after stress. Life Sci 121: 124-134, 2015. 
18. Wang T, Li Y, Wang Y,Zhou R, Ma L, Hao Y, Jin S, Du J, Zhao C, Sun T and Yu J: Lycium barbarum polysaccharide prevents focal cerebral ischemic injury by inhibiting neuronal apoptosis in mice. PLoS One 9: e90780, 2014.

19. Livak KJ and Schmittgen TD: Analysis of relative gene expression data using real-time quantitative PCR and the 2(-Delta Delta C(T)) method. Methods 25: 402-408, 2001.

20. Caltana L, Merelli A, Lazarowski A and Brusco A: Neuronal and glial alterations due to focal cortical hypoxia induced by direct cobalt chloride $(\mathrm{CoCl} 2)$ brain injection. Neurotox Res 15 348-358, 2009.

21. Caltana L, Rutolo D, Nieto ML and Brusco A: Further evidence for the neuroprotective role of oleanolic acid in a model of focal brain hypoxia in rats. Neurochem Int 79: 79-87, 2014.

22. Guan D, Su Y, Li Y, Wu C, Meng Y, Peng X and Cui Y: Tetramethylpyrazine inhibits $\mathrm{CoCl} 2$-induced neurotoxicity through enhancement of Nrf2/GCLc/GSH and suppression of HIF1 $/$ /NOX2/ROS pathways. J Neurochem 134: 551-565, 2015.

23. Dai Y, Li W, Zhong M, Chen J, Liu Y, Cheng Q and Li T: Preconditioning and post-treatment with cobalt chloride in rat model of perinatal hypoxic-ischemic encephalopathy. Brain Dev 36: 228-240, 2014.

24. Pearson JN and Patel M: The role of oxidative stress in organophosphate and nerve agent toxicity. Ann N Y Acad Sci 1378 : 17-24, 2016.

25. Lan AP, Chen J, Chai ZF and Hu Y: The neurotoxicity of iron, copper and cobalt in Parkinson's disease through ROS-mediated mechanisms. Biometals 29: 665-678, 2016.

26. Venkatesan R, Subedi L, Yeo EJ and Kim SY: Lactucopicrin ameliorates oxidative stress mediated by scopolamine-induced neurotoxicity through activation of the NRF2 pathway. Neurochem Int 99: 133-146, 2016.

27. Gao K, Liu M, Cao J, Yao M, Lu Y, Li J, Zhu X, Yang Z and Wen A: Protective effects of Lycium barbarum polysaccharide on 6-OHDA-induced apoptosis in PC12 cells through the ROS-NO pathway. Molecules 20: 293-308, 2014.

28. Zhu X, Hu S, Zhu L, Ding J, Zhou Y and Li G: Effects of Lycium barbarum polysaccharides on oxidative stress in hyperlipidemic mice following chronic composite psychological stress intervention. Mol Med Rep 11: 3445-3450, 2015.

29. Qi B, Ji Q, Wen Y, Liu L, Guo X, Hou G, Wang G and Zhong J: Lycium barbarum polysaccharides protect human lens epithelial cells against oxidative stress-induced apoptosis and senescence. PLoS One 9: e110275, 2014.

30. Rui C, Yuxiang L, Yinju H, Qingluan Z, Yang W, Qipeng Z, Hao W, Lin M, Juan L, Chengjun Z, et al: Protective effects of Lycium barbarum polysaccharide on neonatal rat primary cultured hippocampal neurons injured by oxygen-glucose deprivation and reperfusion. J Mol Histol 43: 535-542, 2012.
31. Li SY, Yang D, Yeung CM, Yu WY, Chang RC, So KF, Wong D and Lo AC: Lycium barbarum polysaccharides reduce neuronal damage, blood-retinal barrier disruption and oxidative stress in retinal ischemia/reperfusion injury. PLoS One 6: e16380, 2011.

32. Vriend J and Reiter RJ: The Keap1-Nrf2-antioxidant response element pathway: A review of its regulation by melatonin and the proteasome. Mol Cell Endocrinol 401: 213-220, 2015.

33. Na HK and Surh YJ: Oncogenic potential of Nrf2 and its principal target protein heme oxygenase-1. Free Radic Biol Med 67: 353-365, 2014.

34. Ollinger R, Yamashita K, Bilban M, Erat A, Kogler P, Thomas M, Csizmadia E, Usheva A, Margreiter R and Bach FH: Bilirubin and biliverdin treatment of atherosclerotic diseases. Cell Cycle 6: 39-43, 2007.

35. Gan L and Johnson JA: Oxidative damage and the Nrf2-ARE pathway in neurodegenerative diseases. Biochim Biophys Acta 1842: 1208-1218, 2014.

36. Narasimhan M, Riar AK, Rathinam ML, Vedpathak D, Henderson G and Mahimainathan L: Hydrogen peroxide responsive miR153 targets Nrf2/ARE cytoprotection in paraquat induced dopaminergic neurotoxicity. Toxicol Lett 228: 179-191, 2014.

37. Park SY, Kim DY, Kang JK, Park G and Choi YW: Involvement of activation of the Nrf2/ARE pathway in protection against 6 -OHDA-induced SH-SY5Y cell death by $\alpha$-iso-cubebenol. Neurotoxicology 44: 160-168, 2014.

38. Ye F, Li X, Li L, Yuan J and Chen J: t-BHQ provides protection against lead neurotoxicity via $\mathrm{Nrf} 2 / \mathrm{HO}-1$ pathway. Oxid Med Cell Longev 2016: 2075915, 2016.

39. Dwivedi S, Rajasekar N, Hanif K, Nath C and Shukla R: Sulforaphane ameliorates okadaic Acid-Induced memory impairment in rats by activating the $\mathrm{Nrf} 2 / \mathrm{HO}-1$ antioxidant pathway. Mol Neurobiol 53: 5310-5323, 2016.

40. Kwon SH, Ma SX, Hwang JY, Lee SY and Jang CG: Involvement of the Nrf2/HO-1 signaling pathway in sulfuretin-induced protection against amyloid beta25-35 neurotoxicity. Neuroscience 304: 14-28, 2015.

41. Yang Y, Li W, Li Y, Wang Q, Gao L and Zhao J: Dietary Lycium barbarum polysaccharide induces Nrf2/ARE pathway and ameliorates insulin resistance induced by high-fat via activation of PI3K/AKT signaling. Oxid Med Cell Longev 2014: 145641, 2014.

42. He M, Pan H, Chang RC, So KF, Brecha NC and Pu M: Activation of the Nrf2/HO-1 antioxidant pathway contributes to the protective effects of Lycium barbarum polysaccharides in the rodent retina after ischemia-reperfusion-induced damage. Plos One 9: e84800, 2014. 